\title{
Lapurdum
}

LAPURDUM Euskal ikerketen aldizkaria | Revue d'études basques |

Revista de estudios vascos | Basque studies review

$3 \mid 1998$

Numéro III

\section{Nouvelles remarques sur haina}

\section{Georges Rebuschi}

URL URL : https://journals.openedition.org/lapurdum/1672

DOI : 10.4000/lapurdum. 1672

ISSN : 1965-0655

Éditeur

IKER

Édition imprimée

Date de publication : 1 octobre 1998

Pagination : $53-75$

ISBN : 2-84127-152-8

ISSN : $1273-3830$

Référence électronique

Georges Rebuschi, « Nouvelles remarques sur haina », Lapurdum [En ligne], 3 | 1998, mis en ligne le 01 septembre 2010, consulté le 24 février 2022. URL : http://journals.openedition.org/lapurdum/1672 DOI : https://doi.org/10.4000/lapurdum.1672

IKER UMR5478 | Rebuschi G. 


\section{Georges REBUSCHI}

Nouvelles remarques sur Haina

Lapurdum 3 [Bayonne]

(1998)

pp. 53-75 


\section{Georges REBUSCHI}

\section{NOUVELLES REMARQUES SUR HAINA}

1. Le présent article se propose de poursuivre, en le corrigeant partiellement, le travail entrepris dans mes 'Remarques sur le pronom haina', dorénavant $R P H$ (Rebuschi 1997). ${ }^{\mathrm{i}}$

Il repose (sans l'épuiser) sur l'analyse d'un corpus complet (sous réserve d'oubli involontaire), l'ensemble des occurrences de haina dans trois traductions labourdines intégrales des Evangiles, celle de Haraneder (1742), celle de Harriet (1855) et celle de Duvoisin (1859-65). ${ }^{\mathrm{ii}}$ Ce corpus est donné en appendice; j'avais le choix entre plusieurs présentations, qui avaient toutes leurs avantages et leurs inconvénients ; j'ai finalement opté pour une numérotation suivie par traducteur, ce qui permettra un renvoi plus rapide à cet appendice dans le corps de ce texte. Outre le fait que ce corpus étant présenté, les spécialistes qui souhaiteraient proposer une autre analyse de haina n'auraient plus besoin de le constituer, il s'avèrera également utile du point de vue des tendances statistiques qui s'y manifestent.

Cette étude est organisée comme suit. Dans la section 2, je reprends une partie de la conclusion de $R P H$, et illustre les contextes dans lesquels haina apparaît. Dans la section 3 , je présente un tableau récapitulatif indiquant les fréquences selon ces contextes, et indique au $\S 4$ ce qui rend haina si original, et apporte deux arguments nouveaux en faveur de l'interprétation de haina comme quantificateur universel. Dans la section 5, j'introduis explicitement quelques outils théoriques qui ont brillé par leur absence dans $R P H$, et qui seront utiles ensuite: en 6 , où j'élimine la double caractérisation sémantique de haina fournie dans $R P H$, en le réduisant à un objet sémantique tout à fait classique pour certains types de contextes, dont les phrases corrélatives, et en 7 , où je montre qu'en dépit des apparences, cette analyse, associée à l'opération de clôture existentielle des occurrences libres devariables est tout à fait compatible avec les propriétés syntaxiques de haina dans les autres contextes.

\section{La conclusion de $R P H$ contenait le passage suivant (p. 76):}

« On peut résumer l'analyse qui a été faite de la distribution syntaxique et de la contribution sémantique du pronom haina en labourdin des XVIIIe et XIXe siècles comme suit: ce pronom est un élément exclusivement anaphorique, qui s'interprète comme une relative libre universelle (et non [pas] définie) dont le prédicat est normalement fourni par une proposition adjointe à celle contenant haina (sinon, il faut aller chercher dans le contexte une proposition «saillante» qui peut fournir le contenu de ce prédicat). Dans le cas usuel, la proposition subordonnée adjointe peut être (i) une protase conditionnelle contenant un indéfini existentiel, un item de polarité négative, voire un indéfini universel, (ii) une pseudo-relative libre construite à l'aide de 
pronoms interrogatifs, en nor (ere) ... bait-, (iii) éventuellement une véritable relative libre en $-n+a$ (mais qui doit alors s'interpréter comme quantifiée universellement plutôt que comme spécifique), ou encore (iv) une relative en zein ... bait-.

«Ce pronom s'oppose au pronom démonstratif hura qui peut [aussi] figurer dans les trois premiers contextes cités à l'instant par le fait qu'il apporte une quantification universelle à la forme logique de la phrase dont il fait partie - au contraire de hura, [... qui peut] reprendre autant des noms propres que des relatives libres spécifiques ou définies. »)

Pour les lecteurs qui n'auraient pas eu accès à $R P H$, voici quelques illustrations, que je tire préférentiellement de Harriet (dorénavant "Hrt") ou, à défaut, de Haraneder ("Hnd") ou Duvoisin ("Duv"), le numéro suivant l'abrégé du nom du traducteur renvoyant à celui de l'appendice:

$\diamond$ Phrases conditionnelles à protase contenant un indéfini existentiel:
Mc 7,11 (Hrt, 13)
Baldin norbaitek baderro aitari edo amari: "[...]", hainak legea bete duke si quelqu'un s'il-dit père-DAT ou mère-DAT h. loi observé AUX
'Si quelqu'un dit à son père ou à sa mère [ ...], il a observé la loi'

$\diamond$ Phrases conditionnelles à protase contenant un item de polarité négative:
Jn 9, 22 (Hnd, 33) ii $^{\mathrm{ii}}$
... elkar hartuak ziren Juduak, baldin nihork aitortzen bazuen
l'un l'aure pris AUX Juifs, si personne disait si-AUX
Jesus zela Kristo, kasatua izan zela haina sinagogatik.
J. qu'il était le Christ, chassé serait il de la sinagogue
'les Juifs avaient décidé entre eux que si quiconque disait que
Jésus était le Christ, il serait chassé de la sinagogue'

$\diamond \mathrm{Ce}$ que j'appelais «protase conditionnelle contenant un indéfini universel» devrait plutôt être appelé protase de phrase «inconditionnelle», selon l'heureuse expression de Zaefferer (1990), puisqu'il s'agit de constructions (au subjonctif en basque) qui parcourent l'ensemble d'un domaine pour conclure qu'aucune restriction ne s'applique qui exclurait un élément ou un autre du prédicat apporté dans l'apodose (la principale, à droite):
Mt 7,24 (Duv, 6)
Edozeinek beraz adi eta bete detzan ene hitz haukiek, haina, n'importe-lequel donc entende et accomplisse AUX (SUBJ) paroles ces, $h$ harriaren gainean bere etxea jarri duen gizon gurbilaren kide eginen dute. pierre-GEN sur sa maison posé AUX homme soigneuX-GEN pair feront AUX 'Ainsi, quiconque écoute ces paroles sera comparé à un homme avisé qui a bâti sa maison sur le roc' - litt. 'que qui que ce soit entende ..., on le comparera...'

$\diamond$ Propositions corrélatives (j'avoue avoir eu tort de rejeter l'emploi de ce terme au profit de la circonlocution qui figure dans la citation ci-dessus : « une pseudo-relative libre construite à l'aide de pronoms interrogatifs, en nor (ere) ... bait-»; la difficulté venait de ce que les corrélatives dont j'avais connaissance servaient à construire l'équivalent de relatives restrictives, qui pouvaient aussi, mais en aucun cas seulement, être interprétées génériquement) ${ }^{\mathrm{v}}$ : 
(4) Mt 19,29 (Hrt, 7)

Eta nork ere utziko baititu bere etxea, edo bere anaiak... ene izena gatik, et qui même laissera AUX sa maison, ou ses frères mon nom pour hainari ordain emanen zaio ehunetan bertze hainbertze. h.-DATIF équivalent il-lui-sera-donné en-cent autre tant 'Et quiconque aura quité sa maison, ou ses frères... à cause de mon nom en recevra le centuple en récompense' - litt. 'et qui laissera..., il lui sera donné.'

$\checkmark$ Propositions relatives libres (ou «sans antécédent») de valeur universelle:

(5) Jn 5,24 (Hrt, 20)

Ene hitza entzuten duenak, eta sinhesten duenak $^{\mathrm{vi}}$ ni egorri nauena baitan, ma parole entend celui-qui, et croit celui-qui moi envoyé celui-qui en hainak badu bizitze betierekoa.

$h$. a vie éternelle

'Celui qui entend ma parole et croit en celui qui m'a envoyé, celui-là a la vie éternelle'

$\diamond$ Haina peut aussi être suivi par une proposition relative en zein ... bait-:

(6) Mt 19,11 (Hrt, 6)

Eztira ere oro gai horren aditzeko, bainan

Ils-ne-sont même tous capables cela-GEN d'entendre, mais

hainak bakarrik, zeinei emana izan baitzaie.

h.-PLUR seulement à-qui donné il-leur-a-été

'Tous ne peuvent pas comprendre cela, mais seulement ceux à qui cela a été donné.'

$\diamond$ Dans toutes les structures qu'on vient d'illustrer, l'«antécédent» de haina (ou la proposition qui donne un contenu à la variable de prédicat que sa traduction sémantique contient - sur ces notions, voir plus loin), appartient à la phrase maximale ou radicale qui contient aussi haina. Mais il était aussi rappelé dans le passage cité que la détermination pouvait être donnée "contextuellement», au sens où elle était apportée par du matériel extérieur à cette phrase radicale. En voici un ex. tiré de Harriet:
Mt 24,46-47 (Hrt, 9)
46Dohatsua da zerbitzari hura, zeina nausiak, datorrenean, heureux est serviteur ce, lequel le-maître en-(re)venant horrela hari dela aurkituko baitu. ${ }^{47}$ Egiaz erraiten darotzuet, ainsi travaillant trouvera AUX en-vérité je-vous-le-dis haina bere ontasun guzien gaineko ezarriko duela. $h$. ses biens tous-GEN dessus mettra AUX 'Heureux ce serviteur que le maître, en rentrant, trouvera travaillant de la sorte. En vérité, je vous le dis, il l'établira sur tous ses biens.'

3. Si l'on ne fait pas de différence entre les deux types de conditionnelles proprement dites (cp. (1) vs. (2)), on peut donc ramener à six grands types les contextes dans lesquels haina apparaissait. Or la fréquence relative de ces contextes est extrêment variée; il est donc utile de récapituler ce que l'on peut retirer des données de l'appendice. Dans le tableau qui suit, j'indique les numéros des ex. concernés à la suite du total du nombre des occurrences et du pourcentage par rapport au nombre total d'occurrences de haina chez chaque traducteur. D'autres propriétés seront discutées 
dans les sections suivantes. ${ }^{\text {vii }}$

(8) Les contextes liés aux occurrences de haina dans les trois textes recensés.

Total des occurrences de haina

Hnd : 40 ; Hrt : 24 ; Duv : 66; total : 130 .

(i)

Constructions corrélatives (en nor ere..., haina)

Hnd: 9 , soit $22,5 \%$ (numéros 2 à $4,6,8$ à $10,22,32$ )

Hrt : 9 , soit $37,5 \%$ (numéros 2 à $5,7,14,17,23,24$ )

Duv: 28 , soit $42,4 \%$ (numéros 1 à 4,8 à 10,12 à 18, 20, 22, 23, 31, $32,34,48$ à $50,55,56,59,60,66)$

Total: 46 , soit $\quad 35,4 \%$

(ii) Relatives libres en haina... zeina...

Hnd : 18, soit $45 \%$ (numéros 5, 7, 12 à 14, 16 à 19, 21, 23, 25, 26, $28,31,34,37,40)$

Hrt : 6 , soit $25 \%$ (numéros $6,8,10,11,16,19$ )

Duv: 18 , soit $27,3 \%$ (numéros 11, 21, 26 à 29, 33, 37, 40 à 43, 45,

Total: 43 , soit $33,1 \%$

(iii) Conditionnelles

Hnd : 4 , soit $10 \%$ (numéros $15,33,35,39$ )

Hrt: 4, soit $16,7 \%$ (numéros $12,13,15,22$ )

Duv: 4 , soit $6 \%$ (numéros 30, 35, 61, 64)

Total: 12 , soit $9,2 \%$

(iv) Reprise de relatives libres en -(e)na

Hnd : 0 , soit $0 \%$

Hrt : 2, soit 8,3\% (numéros 20,21)

Duv: 6 , soit $9,1 \%$ (numéros $5,24,38,39,54,62$ )

Total: 8 , soit $6,1 \%$

(v) Inconditionnelles

Hnd: 0 , soit $0 \%$

Hrt: 0 , soit $0 \%$

Duv: 5 , soit $7,6 \%$ (numéros $6,7,19,25,44$ )

Total: 5 , soit $3,8 \%$

(vi) Reprises contextuelles extra-phrastiques

Hnd: 8 , soit $20 \%$ (numéros $1,11,20,24,27,29,30,38$ )

Hrt : 3, soit $12,5 \%$ (numéros 1, 9, 18)

Duv: 5 , soit $7,6 \%$ (numéros $36,46,47,57,58$ )

Total: 16 , soit $12,3 \%$

\section{Commentaires}

Il est clair que certains chiffres sont trop bas pour que l'on puisse en tirer quoi que ce soit. Il apparaît cependant clairement que les deux structures majeures sont les constructions corrélatives et les relatives libres en haina + zeina, qui représentent chacune environ un tiers de l'ensemble; tout traitement correct de haina doit donc prendre ces deux contextes comme fondamentaux.

Du point de vue diachronique, on remarquera aussi que:

- si l'augmentation des corrélatives associées à haina est manifeste entre le $18 \mathrm{e}$ et le $19 \mathrm{e}$ siècles, un examen général des constructions corrélatives (associées à hura ou à $\varnothing$, i.e. l'absence de tout pronom explicite) dans les trois textes ne révèle en fait rien de 
particulieR ${ }^{\text {viii; }}$

- si l'on regroupe les structures conditionnelles et inconditionnelles, la stabilité est évidente;

- par contre, il y a une baisse manifeste des reprises purement contextuelles (de 20\% au XVIII à $10 \%$ au XIXe, en faisant la moyenne entre les pourcentages de Harriet et de Duvoisin), et une progression tout aussi nette (et corrélée ?) des reprises de relatives libres topicalisées en -(e)na.

4. Explicitions maintenant ce qui fait de haina un pronom si peu ordinaire.

$\checkmark$ Premièrement, il n'est jamais employé déictiquement, au contraire de hura, qui peut se substituer à haina dans tous les contextes sauf celui où ce dernier est suivi d'une relative libre en zeina (cf. (8ii) et (6)) - voir RPH là-dessus). C'est donc un pronom anaphorique pur. ${ }^{\text {ix }}$

$\diamond$ Deuxièmement, il ne reprend jamais de nom propre, ni d'expression nominale définie. ${ }^{\mathrm{x}}$

$\diamond$ Troisièmement, il ne peut jamais être c-commandé par son antécédent (pour la définition de la c-commande, $\mathrm{cf} . R P H$ ) à partir d'une position argumentale. Dans des phrases équivalentes de (9) donc, les options offertes par le labourdin du XVIII et du $\mathrm{XIX}^{\mathrm{e}}$ siècles sont exclusivement les pronoms «ordinaires »: Ø [ou pro], hura et bera.

Quelqu'un [m'a dit qu'il irait au Paradis]

N'essayez pas de convaincre qui que ce soit [qu'il ira au Paradis]

Celui qui croit de telles choses [devrait se demander s'il a de bonnes raisons pour cela]

Si tu rencontres quelqu'un [qui te dit qu'il ira au Paradis...]

$\diamond$ Quatrièmement, les phrases maximales dans lesquelles il apparaît ont, énonciativement, un statut de loi plutôt que de généralisation. Corrélativement, il n'y a pas de cooccurrence entre haina et un adverbe comme 'souvent' ayant portée sur la phrase maximale.

Ce dernier point (qui n'avait pas été noté dans $R P H$ ) est crucial. En effet, une des analyses les plus fréquentes de l'équivalent anglais ou français de conditionnelles du type illustré par (1) supra est de considérer que la quantification qui les caractérise n'est due ni à l'indéfini quelqu'un de la protase, ni au $i l$ de l'apodose: on suppose la présence d'un opérateur (phoniquement invisible) de généricité (rendu licite par le temps-mode-aspect de la proposition principale) qui lie «non-sélectivement» la traduction des deux pronoms en variables, cf:

(10) a Si quelqu'un parle à son chef, il tremble

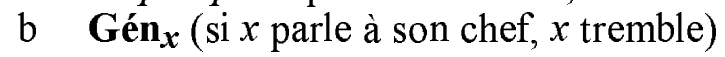

Considérons maintenant (11a), une variante de (10a) introduite par l'adverbe souvent . On note alors clairement que deux interprétations sont disponibles; la première, littérale, est sans grand intérêt pour nous ici; souvent y quantifie des événements : quand Jean parle à son chef, souvent, il tremble, quand Pierre parle à son chef, souvent, il tremble, etc. Ce qui est important, par contre, c'est l'existence de la seconde interprétation, indiquée en (11b) ci-dessous, selon laquelle c'est la variable 
d'individu $\boldsymbol{x}$ qui est quantifiée:

(11) a Souvent, si quelqu'un parle à son chef, il tremble

b $\quad \mathbf{B c p}_{\boldsymbol{x}}$ (si $x$ parle à son chef, $x$ tremble)

(11b), et donc (11a), peuvent se gloser par: Beaucoup de gens tremblent quand ils parlent à leur chef. Or une telle interprétation n'est possible que si les deux pronoms quelqu'un et $i l$ sont effectivement traités comme deux variables liées par un opérateur de quantification dont la source est ailleurs ${ }^{\mathrm{xi}}$. L'absence de telles restrictions adverbiales dans les phrases contenant haina semble donc bien indiquer que la quantification universelle que j'avais attribuée à ces phrases dans $R P H$ provient du mot haina lui-même. On a donc ici un argument de plus corroborant cette hypothèse.

Un autre argument encore peut être ajouté, qui est également fondé sur une absence d'occurrences spécifiques dans le corpus : je n'ai trouvé nulle part d'exemple où une relative libre suivie de guzia 'tout' serait directement reprise par haina (dans le cas de Hnd 30 par ex., la première reprise est par $\varnothing$, si bien que le contexte d'emploi de haina est de type contextuel). Si l'on admet le principe dit d'Interprétation complète (Full Interpretation) de Chomsky (1986), repris sous la forme d'une interdiction de toute quantification à vide (vacous quantification) par les sémanticiens, on voit que ces principes seraient violés si une telle relative libre était corrélée à haina, car la forme logique de la phrase disposerait alors de deux quantificateurs universels pour une seule paire de variables (la variable liée par " $\forall$ " apparaît en effet et dans la restriction, la proposition de gauche, qui contient le prédicat $\mathrm{P}$, et dans la portée nucléaire, la proposition de droite, qui contient le prédicat $Q$ de (12) ci-après.

5.1. Cela dit, la caractérisation sémantique précise de haina ne va pas sans problèmes. Aussi longtemps que l'on se contente d'une formule comme (12), on s'en tient à des banalités difficilement réfutables ${ }^{\mathrm{xii}}$.

(12) haina $\Rightarrow \forall \mathrm{x}[\mathrm{P}(\mathrm{x}): \mathrm{Q}(\mathrm{x})]$

En effet, le principe de compositionalité exige que les aspects logiques du sens soient calculés en fonction non seulement du contenu des morphèmes pertinents, mais aussi de la manière dont ils sont assemblés par la syntaxe ${ }^{\text {xiii }}$. En conséquence, il faut se donner les moyens de décrire précisément, ou de calculer formellement comment on arrive à la construction (de la partie vériconditionnelle) du sens - spécifiquement, la partie droite de (12) où $\mathrm{P}$ et $\mathrm{Q}$ auraient un contenu concret, par ex. 'parler' et 'trembler' respectivement.

5.2. Le traitement standard ${ }^{\mathrm{xiv}}$ est de faire précéder une formule comme la partie droite de (12) par des opérateurs $\lambda$ (lambda) qui transformeront cette formule en prédicat prenant comme argument un prédicat. Commençons par un exemple simple. Si l'on fait abstraction du temps, une phrase comme Jean parle peut se représenter comme en (13), où le prédicat est à gauche, et son argument, entre parenthèses, à droite:

(13) parler(jean) 
Bien entendu, Jean a d'autres propriétés ${ }^{\mathrm{xv}}$; on peut donc faire abstraction du prédicat parler, ce qui donnera (14):

(14) $\lambda$ P.P(jean)

formule qui peut recevoir une interprétation ensembliste : c'est l'ensemble des propriétés de Jean. On revient à (13) en deux étapes. D'abord, si (14) représente un ensemble, cette formule peut aussi se comprendre comme la fonction caractéristique de cet ensemble. En tant que fonction (unaire), elle peut prendre un prédicat comme parler comme argument, ce que note (15):

(15) $[\lambda$ P.P(jean) $]($ parler $)$

(On peut donc lire (15) comme suit: "parler" est un élément de l'ensemble des propriétés de Jean.)

L'opération dite « $\lambda$-conversion» s'appliquera ensuite à (15); elle revient à substituer dans la formule toutes les occurrences de la variable liée par l'opérateur $\lambda$ (ici, l'unique occurrence de $\mathrm{P}$ après " $\lambda \mathrm{P}$ ") par l'argument, provoquant simultanément la disparition de l'opérateur $\lambda$ lui-même. On voit que cette opération donne directement (13).

Considérons maintenant une proposition comme (16):

(16) Quiconque parle tremble

Il n'est pas possible de représenter directement le contenu de cette phrase comme en (13), puisque quiconque est un pronom indéfini, non référentiel. Qui plus est, ce pronom ne peut pas fonctionner seul (*quiconque tremble n'est pas une phrase indépendante bien formée, au contraire de tout le monde tremble). Cet élément va donc devoir se combiner d'abord avec un prédicat, ici parler, pour donner un argument, qui se combinera avec le verbe trembler (le prédicat de la proposition principale), pour donner compositionnellement son sens à la phrase.

Si on laisse de côté la restriction de quiconque aux humains (comparer tout, qui peut quantifier aussi bien des objets que des humains: tout livre, tout linguiste, mais qui prend des noms plutôt que des relatives comme argument servant à restreindre son domaine), on peut donc noter la contribution sémantique de quiconque - et, en anticipant légèrement, de haina-comme en (17):

(17) quiconque/haina $=>\lambda \mathrm{P} \lambda \mathrm{Q} \forall \mathrm{x}[\mathrm{P}(\mathrm{x}): \mathrm{Q}(\mathrm{x})]$

Les opérations de $\lambda$-conversion s'effectuant de l'extérieur vers l'intérieur, les valeurs de $\mathrm{P}$ (parler) et Q (trembler) seront donc données comme en (18):

(18) $[\lambda \mathrm{P}[\lambda \mathrm{Q} \forall \mathrm{x}[\mathrm{P}(\mathrm{x}): \mathrm{Q}(\mathrm{x})]($ trembler $)]]$ (parler)

La première conversion donnera (19a), et la seconde, (19b), qui définit bien la valeur vériconditionnelle de (16):

(19) a $[\lambda \mathrm{Q} \forall \mathrm{x}[\operatorname{parler}(\mathrm{x}): \mathrm{Q}(\mathrm{x})]]($ trembler)

b $\forall \mathrm{x}[\operatorname{parler}(\mathrm{x}): \operatorname{trembler}(\mathrm{x})]$

5.2. Revenons à haina. Ce morphème partage beaucoup de caractéristiques avec quiconque, au moins dans le cas particulier des constructions en haina... zeina. Un 
exemple est fourni en (20), où l'on fera abstraction de la marque non-pertinente d'hypothèse baldin ... ba, 'si':

(20) Jn 10,35 (Duv, 63)

Baldin jainko deitu baditu hainak zeineri ateratu baitzaiote Jainkoaren hitza..

si dieu appelé si-AUX $h$ à-qui sorti qu'il-leur-sont de-Dieu le-mot

'S'il a appelé dieux (tous) ceux à qui la parole de Dieu est venue...'

Le prédicat $\mathrm{P}$ des formules ci-dessus est ici $\lambda y\left[\right.$ venir-à(parole,y) ${ }^{\mathrm{xvi}}$, et c'est lui qui va servir d'argument à la fonction (17). A partir de (21a), on obtient donc (21b), après plusieurs $\lambda$-conversions successives :

$$
\begin{array}{ll}
\mathrm{a} & [\lambda \mathrm{P}[\lambda \mathrm{Q} \forall \mathrm{x}[\mathrm{P}(\mathrm{x}): \mathrm{Q}(\mathrm{x})](\lambda z[\text { appeler(il, z, dieu })])]](\lambda y[\text { venir-à }(\text { parole, } \mathrm{y})]) \\
\mathrm{b} & \forall \mathrm{x}[[\text { venir-à(parole, } \mathrm{x})]:[\operatorname{appeler}(\mathrm{il}, \mathrm{x}, \text { dieu })]]
\end{array}
$$

Tout serait donc d'une simplicité ... biblique, si les relatives en zeina suivaient toujours immédiatement haina. Malheureusement, il n'en est rien : même en incluant les cas où haina n'est séparé de zeina que par baizen, baizik ou bakarrik 'seulement', ces cas de (quasi-)juxtaposition ne représentent respectivement que $61 \%, 33 \%$ et $44 \%$ des occurrences pertinentes dont les références sont données en (8ii). Il faut donc admettre que la séparation de haina et de zeina (typiquement par la copule fléchie, ce qui montre que haina est alors en position focale, comme dans (22) ci-après) est liée à un processus d'extraposition de la relative, extraposition qui laisse une trace adjacente à haina; j'introduis donc cette trace (sous la forme d'un $t$ coïndexé avec la relative) dans l'extrait suivant, donné à titre d'illustration (Duv 41 a pratiquement le même texte):

(22) Lc 8,21 (Hnd, 19)

Ene ama eta ene anaiak, hainak $\boldsymbol{t}_{\boldsymbol{i}}$ dire, [zeinek aditzen baidute [...]

POSS-1 mère et frères, $h$. sont qui entendent AUX

Jainkoaren hitza $]_{i}$

de-Dieu la-parole

'Ma mère et mes frères, ce sont ceux(-là même) qui entendent la parole divine'

Si l'on admet, comme c'est courant aujourd'hui, que les traces qui ne sont pas des traces d'opérateurs sont en fait des éléments dont seule la forme (ou la face) phonique a été déplacée, leur contenu sémantique va être interprété in situ en forme logique: la compositionalité est donc encore respectée, avec haina défini comme quiconque en (17).

6.1. Le cas traité à l'instant n'était pas developpé dans $R P H$, où une formule minimalement distincte de (17) [reprise ici comme (23a)] pour haina était proposée, (34a)-(36a) dans $R P H$, répété ici comme (23b):

$$
\begin{array}{lll}
\mathrm{a} & \text { haina } & =>\lambda \mathrm{P} \lambda \mathrm{Q} \forall \mathrm{x}[\mathrm{P}(\mathrm{x}): \mathrm{Q}(\mathrm{x})] \\
\mathrm{b} & \text { haina } & =>\lambda \mathrm{Q} \lambda \mathrm{P} \forall \mathrm{x}[\mathrm{P}(\mathrm{x}): \mathrm{Q}(\mathrm{x})]
\end{array}
$$

L'inversion des opérateurs $\lambda(\lambda \mathrm{Q}$ précède ici $\lambda \mathrm{P})$ avait le mérite de permettre de traiter directement de cas de figure comme (8i), les corrélatives, (8iii), les conditionnelles, ou (8iv), les relatives libres disloquées. En effet, si l'on part de structures de surface comme (4), (1) ou (5) respectivement, l'interprétation logico-sémantique des phrases 
ne peut se faire compositionnellement que si haina se combine d'abord avec le prédicat sémantiquement pertinent ${ }^{\mathrm{xvii}}$ fourni par la proposition à laquelle il appartient, et ensuite seulement avec le prédicat fourni par la protase.

Mais cette inversion avait aussi un grand défaut, celui de ne correspondre à rien de connu ! ${ }^{\text {xiii }}$ La question se pose donc de savoir si l'on doit permettre à haina d'être doublement caractérisé - soit comme quiconque, par (23a), soit, de manière hautement idiosyncrasique, par (23b). Il n'est évidemment pas de bonne méthode de proposer deux caractérisations si on peut n'en donner qu'une, et il n'est pas non plus très sérieux de se satisfaire d'un résultant surprenant aussi longtemps que l'on n'a pas cherché à voir si la variante plus traditionnelle ne pourrait pas suffire.

Je vais donc essayer de justifier dans cette section l'élimination de (23b), et de montrer comment on peut raisonnablement ramener l'essentiel des usages de haina à (23a).

6.2.1. Considérons d'abord les corrélatives. Comme je l'ai dit plus haut, les corrélatives basques semblent bien ne fournir, en première approximation, que des relatives libres universelles, et non pas des relatives définies - en fait, elles fournissent des prédicats. Cela dit, la forme même des corrélatives est très proche de celle des relatives en zeina. Ainsi, dans le dialecte qui nous intéresse, c'est le même préfixe bait- qui, dans les deux cas, marque la subordination (alors qu'en haut-navarrais, par exemple, la forme verbale fléchie des corrélatives proprement dites est ou était en (e) $\left.n^{\mathrm{xix}}\right)$. En fait, la seule différence est liée au choix de nor dans les corrélatives, et à celui de zein- dans les relatives postposées. J'ai probablement accordé trop d'importance à cette différence dans $R P H$ - et cela, d'autant plus que Duv offre cinq cas de corrélatives introduites par zeina, dans des contextes qui ne sont pas reliés au discours (c'est-à-dire dans des contextes où la quantification se fait sur un ensemble de référence connu des locuteurs). On peut donc simplement considérer que dans les deux cas, le mouvement syntaxique de cet élément, nor ou zein-, revient à construire syntaxiquement l'opérateur $\lambda$ qui lie une variable d'argument et sert donc à caractériser de manière générale les prédicats (voir (21) supra et les notes 16 et 17). En d'autres termes, on peut proposer une traduction ou équivalence directe entre la représentation syntaxique (24a) et la notation logico-sémantique (b) ${ }^{\mathrm{xx}}$.

$$
\begin{aligned}
& \text { a } \left.\left.\quad\left[\mathrm{CP} \text { Nor/zein }{ }_{i} \text { (ere) [C' mintzo bait-da [IP } \boldsymbol{t}_{\boldsymbol{i}} \ldots\right]\right]\right] \\
& \mathrm{b} \quad \lambda \mathrm{x} \quad[\operatorname{mintzo} / \text { parle }(\mathrm{x})]
\end{aligned}
$$

Si l'on admet que lorsqu'une relative est dissociée de haina, c'est suite à un déplacement de la relative vers la droite, on pourra donc admettre que les corrélatives instancient le même type de mouvement, mais vers la gauche.

6.2.2. Qu'un tel mouvement soit syntaxiquement possible est corroboré par le fait même qu'il n'a pas toujours lieu. Si je n'ai pas d'exemple à fournir avec haina luimême, il y en a quelques uns avec hura, dont on sait qu'il pouvait toujours se substituer à haina dans ce contexte ${ }^{\mathrm{xxi}}$. Or il existe des cas où ce que l'on appelle proposition corrélative semble bien être immédiatement adjacent au pronom hura. 
C'est par exemple le cas de (25) ${ }^{\mathrm{xxii}}$.

(25) Luc 10,22 (Hnd)

nihork ez daki nor den Semea Aitak baizen, personne ne sait qui est le-Fils le-Père excepté ez nor den Aita [[Semeak baizen] eta ni qui est le-Père le-Fils excepté et [NORI ERE Semeak agertu nahiko baidu, ETA HARK baizen]]. à-qui même le-Fils révéler voudra bait-AUX et lui excepté 'Nul ne sait qui est le Fils sauf le Père, ni qui est le Père [[sauf le Fils] et [sauf celui à qui le Fils voudra le révéler]]'

Comme l'indique la parenthétisation du texte et de sa traduction en français, il y a coordination de deux syntagmes qui sont composés chacun de baizen 'sauf' précédé, au moins dans le premier cas, d'un syntagme nominal. La question est donc de savoir quelle est la nature de la chaîne (que j'ai mise en italiques) qui va de nori ere à eta hark. Tout semble indiquer qu'il s'agit aussi d'un syntagme nominal, et, en dépit de la présence de eta 'et' en avant-dernière position, d'un syntagme nominal qui, s'il a une structure interne complexe, $n^{\prime} e s t$ pas lui-même construit par coordination de deux syntagmes nominaux distincts. Il est clair en effet que la référence est universelle - ou, pour reprendre les termes de Jacobson employés dans $R P H$, à propos de la sémantique de hura, qu'elle se fait à un individu pluriel maximal (qui ne tolère donc pas d'exceptions).

Avant de poursuivre, soulignons que cette construction, quoique rare, n'est pas un hapax. On la rencontre encore dans la langue contemporaine, ainsi que me l'a confirmé l'académicien Emile Larre. En voici un exemple non construit (en bas-navarrais):

(26) Marc 10,40 (Léon 1946)

toki hoik [[norentzat ere eginak baitira eta heie]nak] dira.

lieu ces pour-qui même faits bait-ils-sont et les-leurs ils-sont

'ces endroits sont ceux de ceux pour qui ils ont été faits'

La corrélation «interrogatif» $\approx$ démonstratif est ici un peu plus complexe, puisqu'elle ne concerne pas des arguments des deux verbes, mais un adverbial ou adjoint destinatif (norentzat) d'abord, puis un génitif qui spécifie un SN sans tête nominale explicite (heien- $\varnothing-a k$ ), mais elle est exactement du même type: entre le sujet toki hoik et le verbe fléchi dira qui clôt le syntagme verbal, il n'y a de place que pour un syntagme en position (et fonction) attributive, cf. toki hoik heienak dira, 'ces endroits sont les leurs', litt. 'ils sont les Ø d'eux'.

6.2.3. Je suis donc fortement enclin à penser que les syntagmes nominaux [nori ere Semeak agertu nahiko baidu, eta hark] de (25) et [norentzat ere eginak baitira eta heie(nak)] de (26) sont en quelque sorte la source syntaxique des constructions corrélatives proprement dites. Partons plus spécifiquement du fait que, dans les constructions corrélatives, le pronom corrélat (que ce soit hura ou haina), est très typiquement en position focale, immédiatement à gauche du verbe et donc en tête de proposition, et aussi que, sauf chez Duvoisin, ce même corrélat (et/ou la proposition de droite) est très souvent introduit par eta (c'est aussi le cas chez Léon 1946). 
Tout cela se met en place si l'on admet une structure de base comme (27):

$$
\text { [X" Corrélative [X' eta [sN pronom]] }
$$

L'objet syntaxique X" est probablement la projection d'une catégorie fonctionnelle audessus d'un $\mathrm{SN}^{\mathrm{xxiii}}$. Adoptant le mécanisme de focalisation décrit par Ortiz de Urbina (1989), entre autres auteurs, on peut considérer que ce X" est en position de spécificateur de $\mathrm{CP}$, et que le verbe auquel il est adjacent est en $\mathrm{C}^{\circ}$. Sauf cas marqué de topicalisation par dislocation, le X" est donc en position initiale dans le CP, et la corrélative n'a même pas besoin d'être extraite pour s'adjoindre à gauche de ce $\mathrm{CP}$ : dans la position qu'elle occupe, quand le X" est focalisé, elle occupe déjà une position initiale.

Mais, bien entendu, le déplacement du corrélat vers Spéc,CP n'est pas obligatoire. S'il n'y a pas mouvement, la corrélative aurait tendance à monter s'ajoindre à gauche du $\mathrm{CP}$ d'origine, quoique, comme le montrent les ex. (25) et (26), cela ne soit pas obligatoire - en fait, la très classique contrainte des NP coordonnés de Ross interdit même l'extraction hors d'un syntagme coordonné à une autre; on pourrait donc admettre que c'est cette contrainte qui bloque le déplacement dans (25), si l'on adopte une structure de type (27) pour coder un contenu qui peut l'être autrement (cf. la note 22).

6.2.4. Ce qui précède est évidemment très hypothétique, de nombreux problèmes restant à résoudre, notamment la présence (rare il est vrai) de eta à gauche de la proposition principale lorsque hura ou haina n'est pas en position focale, ou lorsque le corrélat n'est pas réalisé phoniquement (Ø ou pro). L'approche suggérée à l'instant a cependant le mérite d'être articulable à l'analyse des constructions en haina ... zeina : en effet, on peut penser que (27) n'est pas la structure réelle, laquelle serait plus riche, cf. (28):

\section{[X" Corrélative $i\left[\mathrm{X}^{\prime}\right.$ eta $\left[\mathrm{sN}\right.$ pronom $\left.\left.\boldsymbol{e}_{i}\right]\right]$}

où $\boldsymbol{e}$ est une position vide liée à la corrélative. L'idéal serait évidemment de pouvoir dire que ce $\boldsymbol{e}$ est la trace d'un déplacement (rendu possible par la présence de la tête fonctionnelle eta?): on aurait alors une structure presque exactement symétrique de celle postulée plus haut pour les relatives extraposées en zeina avec une trace à nouveau interprétée directement in situ comme contenant le prédicat qui se combine sémantiquement avec haina ou hura (cf. la trace $\boldsymbol{t}$ dans (22)).

En résumé, la structure de base serait constituée d'un pronom originellement suivi d'une relative adjointe au SN dominant ce pronom. S'il n'y a pas plus de structure, la relative pourra être extraposée (vers la doite), cf. (22). Mais si le SN complexe ainsi constitué est structuralement le complément d'une tête fonctionnelle (qui sera phoniquement réalisée par eta ou n'aura pas de réalisation phonique), la relative effectuera un premier déplacement vers le spécificateur de cette tête fonctionnelle, et, le cas échéant, montera ensuite s'adjoindre à $\mathrm{CP}$. Du point de vue sémantique, par contre, tout se passera comme s'il n'y avait pas eu de mouvement : la relative sera interprétée comme la propriété différentielle restreignant le domaine quantifié - par 
" $\forall$ " dans le cas de haina, et par "l" (iota) dans le cas de hura (sur ce point, cf. $R P H$ ), ce qui permet, au moins dans le cas des corrélatives, d'éliminer la caractérisation (23b) au profit de la seule définition (23a).

6.3. Le cas des relatives libres disloquées en -(e)na peut sembler plus complexe du point de vue de la compositionalité, dans la mesure où une relative libre est typiquement interprétée comme un argument (techniquement, comme une "entité» si elle est spécifique, ou comme un «quantificateur généralisé» [QG] si elle est universelle). Noter cependant qu'il y a dix ans déjà, Partee (1987) proposait que le changement de type sémantique d'une expression nominale (entité, QG) en un prédicat, quoique marqué, soit reconnu comme nécessaire. Qui plus est, le basque requiert indépendamment que les relatives en -(e)na soit parfois interprétées comme des propriétés et non comme des entités ou des QG. On sait en effet que la distinction sémantique entre relatives restrictives et relatives appositives n'est pas marquée comme telle en basque (cf. Oyharçabal 1985, 1987): les relatives qui précèdent le nom-tête peuvent en effet être interprétées des deux manières, et il en va de même des trois types de relatives qui suivent ce nom tête, qu'elles soient (i) introduites par zeina + bait-, (ii) introduites directement par bait-, ou encore (iii) qu'elles aient la forme d'une relative libre, comme dans l'ex. suivant, (Oyharçabal 1987, p. 180) xxiv. $^{\text {xiv }}$

$$
\begin{aligned}
& \text { etorriko da egun.A, [orok igurikatzen dugu.N.A] } \\
& \text { venir-fut AUX jour-SG-ABS tous attendant, AUX-N-Ø-SG-ABS } \\
& \text { 'Le jour que nous attendons tous viendra' }
\end{aligned}
$$

Pour que (29) reçoive l'interprétation proposée, il faut que la chaîne entre crochets ne soit pas interprétée comme dénotant une entité, mais une propriété qui participera d'une manière ou d'une autre à la détermination de l'antécédent, en permettant de construire l'intersection de l'ensemble des \{jours\} et de l'ensemble des \{objets-quenous-attendons $\}$ (intersection qui ne possède, au singulier, qu'un seul élément). On peut adopter par exemple la théorie de Bach \& Cooper (1978) pour établir un rapport sémantique correct entre un antécédent doté de son spécificateur, et une relative adjointe à cet antécédent: comme on l'a dit, la contribution de Partee, quant à elle, concerne l'interprétation de la relative libre comme propriété.

Si l'on revient à (5) / (8iv), la structure qui nous intéresse ici, on voit donc que la grammaire du basque permet, et même exige, indépendamment de cette structure, que certains objets qui ont la forme d'une relative libre soient interprétés comme des prédicats (ou propriétés). En généralisant la partie pertinente de (28), à savoir que haina est toujours associé à une place vide interprétable comme une propriété, on voit que le cas de figure ici décrit n'est qu'un cas particulier du calcul compositionnel plus général de la contribution sémantique de haina à la phrase à laquelle il appartient. (Ajoutons que si ce qui précède est correct, le curieux ex. Hnd 37, où haina, au lieu d'être suivi par une relative en zein, est suivi d'une relative en -(e)na, devient en fait tout naturel ${ }^{\mathrm{xxv}}$.)

7.1. La section 6 a réglé $73 \%$ des cas de figure: (8i), (8ii) et (8iv) (95 des 130 
occurrences recensées) ${ }^{\text {xxvi }}$. Un gros quart subsiste donc encore. La question est de savoir si les $27 \%$ restants justifient le maintien de (23b) comme alternative à (23a). En fait, il n'en est rien, car le cas de figure représenté par (7) / (8vi), c'est-à-dire celui des reprises contextuelles extra-phrastiques, ne peut de toute manière pas être traité de la sorte. Ce dont on a besoin ici, c'est d'admettre qu'en l'absence d'un prédicat permettant d'interpréter la place vide associée à haina, la variable de prédicat $\mathrm{P}$ de (23a) fait l'objet d'une clôture existentielle ${ }^{\text {xxvii; }}$, en d'autres termes, l'absence d'argument pour l'expression- $\lambda$ globale de (23a) conduit à la réécriture (30):

(30) $\lambda \mathrm{Q} \exists \mathrm{P} \forall \mathrm{x}[\mathrm{P}(\mathrm{x}): \mathrm{Q}(\mathrm{x})]$

Le haina de (7) par exemple s'interprètera comme 'une telle personne', ou mieux, 'toute personne (qui est) telle', où 'tel(le)' marque une propriété différentielle non-explicite mais bien réelle : ce qui revient à dire que pour comprendre la phrase, il faut chercher un prédicat «saillant» dans le contexte; dans le cas de (7), c'est un prédicat complexe, '(être) un serviteur que le maître trouvera au travail en rentrant'.

7.2. Que peut-on dire maintenant des conditionnelles et inconditionnelles (8iii) et (8v) ? Le mécanisme décrit à l'instant suffit; en effet, si la subordonnée conditionnante, ou protase, peut difficilement être interprétée comme un prédicat, elle contient bien un prédicat, prédicat qui va de plus être naturellement «saillant» par rapport à la conditionnée qui suit (l'apodose). Autrement dit, comme il n'y a pas de prédicat pouvant servir d'argument à la $\lambda$-expression de (23a dans les phrases conditionnelles, la règle de clôture existentielle qui donne (30) s'applique, tandis que la subordonnée à gauche, qui contient un prédicat saillant fournit ce prédicat pour l'interprétation. Considérons l'ex. (1). L'argument de (30) est le prédicat 'observer la loi'; par $\lambda$ conversion, on obtient:

(31) $\exists \mathrm{P} \forall \mathrm{x}[\mathrm{P}(\mathrm{x}):$ observer(x,la loi) $]$

Autrement dit, (31) est l'interprétation de la proposition principale, ou conditionnée, de (1), disons, 'toute personne (qui est) telle respecte la loi'. La conditionnante, de son côté, contient un prédicat, 'dire ceci'.

On obtient donc une forme logique qui correspond à la glose (32) ${ }^{\text {xxviii. }}$

(32) SI [il existe une personne $\mathrm{x}_{1}$ qui dit ceci], ALORS [il existe une propriété $\mathrm{P}$ telle que toute personne $\mathrm{x}_{2}$ qui a la propriété $\mathrm{P}$ observe la loi]

'Dire ceci' étant contextuellement saillant va donc être interprété comme le contenu de P, sans plus:

(33) SI [il existe une personne $x_{i}$ qui dit ceci],

ALORS [toute personne $x_{j}$ qui dit ceci observe la loi]

qui, quoique semble lourd, respecte trivialement les conditions de vérité de la seule conséquente ${ }^{\mathrm{xxix}}$.

8.1.En résumé, on a réglé à un détail près l'essentiel des occurrences de haina dans le corpus, celles où cet élément apparaît superficiellement comme un pronom: c'est un 
pronom qui ne réfère pas, mais quantifie universellement un domaine défini par une propriété qui est ou bien apportée par une relative (éventuellement corrélative), ou bien est contextuellement saillante. Restent, d'une part, l'unique cas où haina est suivi de hura (Hnd, 36), attesté par ailleurs selon le $D G V$, et sur lequel je n'ai rien à dire, et, d'autre part, les quatre cas où haina suit un nom et fonctionne donc comme déterminant (Hnd 17, 21, 25 et Hrt 16) ${ }^{\mathrm{xxx}}$. Structuralement, il est parfaitement possible que haina soit à considérer comme un déterminant typiquement intransitif, c'est-à-dire ne prenant usuellement pas de SN comme complément sur sa gauche, mais pouvant marginalement le faire; du point de vue sémantique, ce nom apporte une restriction supplémentaire au domaine quantifié universellement, mais l'exigence d'une position vide liée par une relative interprétée comme une propriété reste manifeste: tous les ex. de ce types sont en effet construits avec une relative en zeina à droite; il n'y a donc rien de vraiment particulier à dire de ce côté.

8.2. Le « détail» auquel je viens de faire allusion concerne l'existence de cas, rares sauf chez Duvoisin (aucun chez Harriet, et un seul chez Harander), où le référent de haina est singulier. Tous ces cas ne sont pas, en toute logique, incompatibles avec l'analyse quantificationnelle de ce morphème: les logiciens parleraient simplement de "quantification dégénérée», car aucun principe ne peut interdire de quantifier universellement un singleton. La rareté des occurrences est alors peut-être stylistique, en ce sens qu'inconsciemment, l'emploi de cet outil a pu être ressenti comme ce que l'on appellerait aujourd'hui humoristiquement l'utilisation d'un bazooka pour tuer un mouche... ${ }^{\mathrm{xxi}}$

Cela dit, il faut distinguer entre le cas général de quantification dégénérée, et un cas très particulier de reprise contextuelle. L'hypothèse que haina se traduit par une quantification universelle est encore défendable, donc, dans des cas comme Duv, 54 (relative libre en -(e)na disloquée: 'celui qui m'a envoyé'), 55 (corrélative: 'celui qui m'a guéri'), ou 66 (relative en zeina: 'celui auquel j'offrirai le pain trempé'), puisqu'il est possible de construire un ensemble de référence à quantifier - comme on le voit en français même, si l'on fait abstraction de ce que l'on sait par ailleurs, ces expressions sont ambiguës et pourraient dans d'autres contextes renvoyer à des ensembles contenant plusieurs éléments.

Par contre, dans les trois cas suivants: Hnd 24 et Duv 47 (Luc 17,16) et Duv 36_(Marc 14,21), la situation est plus sérieuse, puisqu'un individu (une entité) et un seul est désigné dans le verset précédent; il n'est donc plus possible de quantifier sur un singleton, et haina doit être alors considéré comme un strict équivalent du démonstratif hura, de manière analogue à l'ex. (4) de $R P H$. 
${ }^{\mathrm{i}}$ La rédaction de $R P H$ a été suivie de présentations orales progressivement modifiées au séminaire du groupe de syntaxe du LANDISCO de l'Univ. de Nancy 2 (janvier 1998), et au séminaire de syntaxe avancée de Paris 8 (janvier et mars 1998), ainsi que sous forme de communication au Colloque de sémantique non-lexicale de Paris 7 (mai 1998), cf. Rebuschi (1998); je remercie les diverses audiences pour leurs critiques et leurs remarques, qui sont en partie à l'origine de ce travailci, ainsi que la Biblioteka Azkue, qui m'a procuré une copie de l'intégralité de Harriet (1855).

${ }^{\text {ii }}$ La traduction de Harriet, en dépit de son titre, se limite aux quatre Evangiles; comme je tenais à comparer systématiquement les données pertinentes, je me suis donc borné au texte le plus court (on sait que Haraneder a traduit l'ensemble du Nouveau Testament - bien qu'à l'inverse de Harriet, il ait intitulé son texte J.C.en Evangelio Saindua! -, et Duvoisin, la Bible entière). On peut noter au passage que je ne disposais pas du texte de Harriet il y a un an, mais que ce dernier n'apporte auncun contre-exemple aux résultats obtenus sur la base des deux autres traductions ainsi que sur l'Imitation traduite par Chourio, citée dans $R P H$ - au contraire, les passages faisant difficulté pour l'analyse que je propose y sont construits sans haina. (Qu'il me soit permis au passage de rappeler à certains critiques, mécontents de ne me voir utiliser que des traductions, qu'il existe aucun élément lexical ou grammatical équivalent de haina dans leurs textes de départ, qu'il s'agisse du latin ou du français.

iii Duv (61) a une traduction proche, avec haina également; quant à Harriet, il n'emploie apparemment jamais haina dans de tels contextes; voici sa trad. du même verset (où le symbole ' $\emptyset$ ' indique qu'il n'y a pas de pronom explicite) : ...ezen elkar hitz hartuak ziren Juduek, baldin eta nehork aitortzen bazuen Jesus Kristo zela, izanen zela Ø etxatua batzarretik.

${ }^{\text {iv }}$ On ne trouve pas ces constructions chez Harriet, ni dans les Evangiles de Haraneder, mais ce dernier en offre au moins un ex. dans les Epîtres $(\mathrm{Jc} 1,25)$ - ce qui montre bien les difficultés qu'il y a à travailler sur corpus.

${ }^{v}$ Voir par ex. Cooper (1979) pour le hittite, Keenan (1985) ou Dayal (1997) pour le hindi, ou encore Zribi-Hertz (1995) pour le bambara. Depuis, j'ai pris connaissance des corrélatives dans les langues slaves, qui se comportent de manière identique à celles du basque (Izvorski 1996, Boskovic 1997)

${ }^{\text {vi }}$ Voir la note 25 sur le fait que ce qui apparaît comme deux relatives libres coordonnées, et semble donc renvoyer à deux (types d') individus, ne réfère souvent qu'à un seul et unique individu ou type.

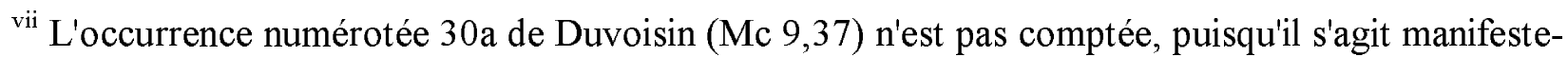
ment d'une coquille; les deux autres trad. ont bien, comme on peut s'y attendre, zeina: Nausia, ikusi dugu gizon bat, zure izenean deabruak kentzen dituena, zeina ez baida gureetarik... (Hnd); Ikusi dugu norbait zure izenean deabruak egozten zituena, zeina ezpaitabila gurekin... (Hrt).

viii Rappelons que si haina a disparu, les corrélatives elles-mêmes sont encore bien vivantes non seulement en labourdin, mais aussi en bas-navarrais.

${ }^{\text {ix }}$ Les passages des Evangiles où des démonstratifs sont employés déictiquement (il s'agit bien entendu de dialogues) ne manquent pourtant pas. Par ailleurs, lorsque hura est effectivement suivi d'une relative en zeina, il est précédé d'un nom et fonctionne donc comme déterminant, et non comme pronom (au sens de pro-SN). 
${ }^{\mathrm{x}}$ «Expressions nominales» au sens strict, avec un nom-tête lexical; comparer le cas (5)/(8iv). Noter deux exceptions: Hnd 1, que Hrt 1 s'est hâté de corriger non pas en supprimant haina, mais en supprimant le nom-tête de l'antécédent, et Hrt 15 (où le SN est malgré tout enchâssé dans une protase conditionnelle).

${ }^{x i}$ Considérons à ce titre Mt 19,30: la Vulgate a Multi 'beaucoup de gens', et nos trois traducteurs, quantifient l'argument sujet avec hainitz (Hnd, Hrt) ou asko (Duv), 'beaucoup' dans les deux cas. Si le mécanisme décrit à l'instant n'était pas très naturel, on ne pourrait comprendre pourquoi Léon (1946) a, lui, opté pour la variante suivante : Maizenik lehenak dira azken eta azkenak lehen, litt. 'Souvent, les premiers sont derniers et les derniers, premiers'.

${ }^{x i i} \mathrm{Cf}$. (16) dans $R P H$, où une coquille a malheureusement fait sauter le quantificateur ' $\forall$ '

xiii C'est évidemment le prix à payer si l'on refuse le holisme sémantique de Wittgenstein, Quine, ou Chomsky.

xiv Je prie les sémanticiens de bien vouloir ou bien sauter cette sous-section, ou bien me pardonner les simplifications et raccourcis que je me permets d'y faire.

${ }^{\mathrm{xv}}$ Le terme de «propriété » s'entend ici au sens large, et ne recouvre donc pas seulement des propriétés stables, mais aussi des activités (définitoires ou non)

${ }^{\text {xvi }}$ L'abstraction est ici faite sur un argument, complication nécessaire due au fait que le prédicat $P$ qui permet d'identifier ceux à qui s'applique le prédicat Q 'être appelés dieux' n'est pas le sujet syntaxique de la relative. En fait, il en va de même de Q, qui est plus exactement:

$\lambda x[a p p e l e r(i 1, x, d i e u)]$ puisque la principale n'est pas au passif. Pour prendre un cas maximalement simple, l'abstraction sur l'argument, et non plus le prédicat, dans (13), donnerait (i), la fonction caractéristique de l'emsemble des individus qui parlent / ont la propriété de parler. La réintégration du sujet s'effectuerait donc à partir de (ii), par $\lambda$-conversion :

(i) $\lambda x[\operatorname{parler}(\mathrm{x})]$

(ii) $[\lambda x[\operatorname{parler}(\mathrm{x})]](j e a n)$

xvii Voir la note précédente. Dans le cas de (4) par exemple, le prédicat sémantique n'est pas le prédicat fourni sur la base de la dichotomie syntaxique traditionnelle syntagme nominal sujet / syntagme verbal, puisque haina est au datif.

xviii Je remercie Iléana Comorovski qui m'en a fait la remarque.

${ }^{\text {xix }}$ Voir par ex. Lizarraga (1868). Voici un des dix ex. que j'ai pu y relever:

(i) Jn 12,47 Eta nork ere aditzen tuen nere itzak, nik eztut ura juzgatzen. et qui même entend AUX+en mes paroles, moi, je ne juge pas celui-là.

${ }^{\mathrm{xx}}$ Je garde pour plus tard une étude de l'opposition distributionnelle entre nor et zein, ainsi que du fait que ere 'même/aussi' n'apparaît que dans les protases corrélatives; remarquer en particulier que nor peut représenter un parcours sur un ensemble fini et connu des locuteurs (cf. Hnd 22 ou Hrt 17), et qu'inversement zein peut «quantifier» un ensemble ouvert (Duv, 4 ou 8). Noter toutefois que si nor est indéfini, et entraîne donc un accord verbo-nominal par défaut en $3 \mathrm{sg}$., zein-relatif peut au contraire varier en nombre; l'identification «forte» que Chomsky (1986) proposait comme contrainte entre un antécédent et un pronom relatif est alors peut-être le facteur qui détermine la 
sélection de ce dernier plutôt que de nor dans les relatives qui suivent le nom (voir cependant le traitement des corrélatives qui suit immédiatement, qui affaiblit cet argument).

${ }^{\text {xxi }}$ Voir $R P H$ et, ici même, dans l'Appendice, Hrt 5 ou Duv 15.

xxii Pour le même verset, Harriet a une relative libre en -(e)na, et Duvoisin, une construction en haina zeina (cf. Duv 42 dans l'Appendice).

xxiii Les corrélatives du hindi peuvent occuper linéairement la même place : adjonction à gauche du $\mathrm{SN} /$ pronom de reprise (selon Dayal 1997) - quoique la tête $\mathrm{X}^{\circ}$ que je suppose ici (eta) ne soit pas phoniquement réalisée dans cette langue. Noter que s'il n'y a pas de tête fonctionnelle, et donc de structure X-barre, il faudrait postuler une adjonction à un argument, ce qui est en général considéré comme impossible.

xxiv Je laisse de côté les relatives où le nom tête $n u$ est suivi d'une relative en -(e)n... (voir à nouveau les travaux d'Oyharçabal à ce sujet) : on en trouvera ici un exemple dans Duv, 7 ([/gizon zoro, legarraren gainean etxea egiten du]ena] : litt. '[[homme stupide, sable dessus maison fait AUX] -enABS.SG.), 'un/l'homme insensé qui construit sa maison sur du sable', mais elles ne posent, du point de vue interprétatif, aucun problème particulier par rapport aux relatives en -(e)n qui précèdent le nom-tête.

${ }^{x x v}$ De la même manière, on voit qu'une extension de ce raisonnement devrait conduire à une analyse somme toute naturelle du fait que la coordination de deux objets qui ont la forme de deux relatives libres, chacune étant au singulier, permet de construire une intersection-singleton, comme dans (5) supra (cf. aussi Hnd, 29 et bien d'autres exemples), où la lecture littérale, 'celui qui entend ma parole et celui qui croit...' est à la fois contraire au sens, et en contradiction avec le singulier du corrélat. ${ }^{\text {xxvi }}$ Voir $\S 8$ pour une brève discussion des antécédents spécifiques.

xxvii Le mécanisme de clôture existentielle par défaut est dû à Heim (1982: pp. 138 et sv.). Elle le proposait uniquement pour des arguments, mais je ne vois pas pourquoi on ne pourrait pas l'étendre aux prédicats, puisque, de toute manière, la logique du premier ordre est insuffisante pour rendre compte des propriétés sémantiques des langues naturelles.

xxvii Je ne donne pas de formule, car, dans la mesure où il n'y a pas de quantification ou de liage d'une une variable d'argument (ordinaire) dans les deux propositions, il faudrait introduire une variable d'événement supplémentaire dans la restriction et la portée nucléaire.

${ }^{\text {xxix }}$ L'analyse présentée à l'instant pour les conditionnelles a malgré tout au moins un un petit défaut : on devrait pouvoir choisir un prédicat extérieur à la phrase maximale comme prédicat saillant donnant sa valeur à $\mathrm{P}$, or je n'ai jamais trouvé d'exemples de haina «passant par-dessus » le prédicat de la protase pour aller en choisir un plus loin dans le texte...

${ }^{\mathrm{xxx}}$ Le cas est donc moins anecdotique que je ne le laissais entendre dans $R P H$, note 9 .

${ }^{\text {xxi }}$ Par contre, en tout état de cause, il y avait une erreur dans $R P H$, section $6.3(\mathrm{p} .74)$ : le recours à la caractérisation d'un référent en intension ou compréhension ne saurait être confondu avec l'emploi proprement prédicationnel d'un syntagme nominal, emploi illustré en (33c). L'exemple (31) de $R P H$, qui correspond à Duv 26 dans l'appendice à ce texte-ci, tout comme Duv 52, Duv 53 ou Duv 56, relève tout simplement de la quantification dégénérée. 
\& UPRESA D'Etudes basques

\section{Appendice}

\section{Haraneder}

$\mathbf{1}-\mathrm{Mt}, 6 .{ }^{2}$ Noiz ere eginen baiduzu amoina, ez dezazula joaraz tronpeta zure aitzinean, hipokritek egiten duten bezala, zinagogetan eta karriketan gizonez ohoratuak izatea gatik. Erraten darotzuet egia: izatu dute hainek bere golardoa. - (cf. Hrt, 1)

2, 3 - Mt 10, ${ }^{37}$ Nork ere maiteago baidu bere aita edo bere ama ni baino, eta haina ez da ona enetzat; eta nork maiteago baidu bere semea edo bere alaba ni baino, haina ez da gai enetzat. - (cf. Hrt 2, 3 \& Duv, 9)

4 - Mt $15,5 \ldots$ Nork ere erraten baidio bere aitari edo amari : "[...]", 6 egon ahal diteke haina bere aita edo ama lagundu gabe. (cf. Hrt, $4 \&$ Duv, 14)

5 - Mt $19,{ }^{11} \mathrm{Ez}$ dire guziak gai hortakotz, hainak baizen, zeinei eman izan baitzaie zerutik. - (cf. Hrt, 6)

6 - Mt 19, ${ }^{29}$ Eta nork ere utziko baiditu bere etxea edo bere anaiak ... ene izena gatik, hainak izanen duela ehunetan hainbertze... - (cf. Hrt, $7 \&$ Duv, 20)

7 - Mt $20,{ }^{23}$ ordean ene eskuinean edo ezkerrean jartzea ez da ene baitan zuei ematea, bainan hainentzat izanen da, zeinei preparatu baidaroe ene Aitak. - (cf. Hrt, 8 \& Duv, 21)

8, 9 - Mt 20, ${ }^{26}$ Bainan nork ere nahiko baidu izan zuen artean handien, izan bedi haina zuen zerbitzari; ${ }^{27}$ eta nork ere nahiko baidu izan zuen artean lehenbiziko, izan bedi haina zuen sehi.

10 - Mt 21, ${ }^{44}$ Harri haren gainerat eroriko dena hautsiko da; eta nori ere eroriko baitzaio berriz hura gainerat, eta haina lehertuko du. - (cf. Duv, 22)

11 - Mt 24, ${ }^{46}$ Dohatsua diteke zerbitzari hura, zeina aurkituko baidu bere nausiak horla hari dela. ${ }^{47}$ Erraten darotzuet egia, haina bere ontasun guzien gainean ibeniko duela. - (cf. Hrt, 9)

12 - Mc 4, ${ }^{16}$ Dire halaber leku harritsuetarat hazia errezibitzen dutenak hainak, zeinek entzutean hitza, berehala bozkariorekien hartzen baidute... - (cf. Duv, 27)

13 - Mc 4, ${ }^{18}$ Dire berriz elhorriperat hazia errezibitzen dutenak hainak, zeinek entzuten baidute hitza... - (cf. Hrt, $10 \&$ Duv, 28)
$14-\mathrm{Mc} 4,{ }^{20}$ Finean lur onerat hazia hartzen dutenak dire hainak, zeinek entzuten baidute hitza eta errezibitzen ... - (cf. Hrt, $11 \&$ Duv, 29)

15 - Mc 9, ${ }^{41}$ Eta baldin nihork eskandalisatzen badu ene baitan sinhesten duten xume hautarik bat, hobe luke hainak errota harri bat lepoan amarraturik, etxa lezaten itsasoan behera. - (cf. Duv, 32)

16 - Mc 10, ${ }^{40}$ Ordean ene eskuineko edo ezkerreko aldean jartzea, ez dagot niri zuei ematea, bainan hainei emanen zaie, zeinei preparatua izan baitzaie. - (cf. Duv, 33)

17 - Mc $14,{ }^{21}[\ldots]$ bainan zorigaitz gizon hainarentzat, zeinetaz Gizonaren Semea izanen baida traditua; hobe liteke gizon harentzat egundaino sortu ez balitz. - (cf. Duv, 36)

18 - Lc 7,43 ( ${ }^{42}$ Zure ustez zeinek du maite gehienik?) ${ }^{43}$ Ene ustez, hainak, zeinari gehiago baidio barkatu.

19 - Lc $8,{ }^{21}$ Ene ama eta ene anaiak, hainak dire, zeinek aditzen baidute eta obretan ematen Jainkoaren hitza. - (cf. Duv, 41)

20 - Lc $12,{ }^{43}$ Dohatsua diteke zerbitzaria, nausiak datorkenean horla hari dela aurkituko duena. ${ }^{44} \mathrm{Zin}$ zinez darotzuet erraten: dituen ontasun guzien gainean ezarriko duela haina.

21 - Lc 12, ${ }^{47}$ Zerbitzari haina, bada, zeinak jakin baidu nausiaren borondatea eta ez baida egon prestatua eta ez baidu egin hark nahi zuen bezala, zehatua izanen da garrazki. (cf. Hrt, 16)

22 - Lc 14, ${ }^{33}$ Zuetarik nork ere ez baidaroe uko egiten guziei, haina ezin izan daiteke ene diszipulu. - (cf. Har, 17)

23 - Lc 17, ${ }^{1}$ Inposible da ez datozen eskandalak; bainan zorigaitz hainarentzat, zeinez etortzen baidire. ${ }^{2}$ Hobe lizate harentzat eman lekioen errota harri bat lepotik eta artik lezaten itsasoan behera, ezen-ez eskandaliza dezan xume hautarik bat. - (cf. Hrt, 18 \& Duv, 45-46)

24 - Lc 17, ${ }^{15}$ Hetarik bat, ikusi zuenean garbitu zela, bihurtu izan zen oihugoraz Jainkoa laudatzen zuela. ${ }^{16}$ Eta artiki zen ahuspez Jesusen oinetarat eskerrak bihurtzen ziotzala; eta haina zen samaritanoa. - (cf. Duv, 47)

25 - Lc $22,{ }^{22}$ bainan zorigaitz gizon hainarentzat, zeinetaz izanen baida trahitua. 
26 - Jn 1, ${ }^{12}$ Bainan errezebitu izan duten guziei eman izan daroe boterea Jainkoaren haur izateko, hainei zeinek sinhesten baidute haren izenean. - (cf. Hrt, $19 \&$ Duv, 5051))

27 - Jn 3, ${ }^{16}$ Halako maneraz ezen Jainkoak maitatu izan du mundua, non eman izan baidu bere seme bakotxa, haren baitan sinhesten duena gal ez dadintzat; aitzitik izan dezantzat hainak bizitze eternala.

28 - Jn $3,{ }^{29}$ Esposa da haina zeinaren baida esposa..

29 - Jn 5, ${ }^{24}$ Zin zinez dartotzuet erraten, ene hitza entzuten duenak eta ni igorri izan nauena sinhesten duenak, baduela bizitze eternala; eta haina ez da erortzen kondenazionean, aitzitik iragana diteke heriotzetik bizitzerat. - (cf. Hrt, 20)

30 - Jn $6,{ }^{40}$ Semea ikusten duen guziak eta haren baitan duenak sinhesten, izan dezan bizitze eternala, eta nik pitzberrituko dut haina azkeneko egunean.

31 - Jn 6, ${ }^{46} \mathrm{Ez}$ Aita ikusi duelakoan nihork, hainak baizen zeina heldu baida Jainkoa ganik; ezen hura da Aita ikusi izan duena.

32 - Jn $7,18 \ldots$ bainan nork ere bilhatzen baidu hura igorri izan duenaren gloria, eta hura da egiatia eta injustiziarik hainaren baitan ez da. - (cf. Duv, 59)

33 - Jn 9, 22 ... elkar hartuak ziren Juduak, baldin nihork aitortzen bazuen Jesus zela Kristo, kasatua izan zela haina sinagogatik. - (cf. Duv, 61)

34 - Jn 10, ${ }^{35}$ Baldinetariak hainak jainko deitzen baditu, zeinei Jainkoa mintzo baitzitzaien... - (cf. Duv, 63)

35 - Jn $12,26 \ldots$ Baldin nihork ni zerbitzatzen banau, ohoratuko du haina ene Aitak. - (cf. Hrt, 22 \& Duv, 64)

36, 37 - Jn 13, ${ }^{24} \ldots$ Nortaz mintzo ote da? 25 ... Jauna, nor ote da haina hura?

26Ihardetsi zioen Jesusek, "Haina da nik ogia bustirik emanen diokedana" - (cf. Duv, 65)

38 - Jn $14,{ }^{21} \ldots$ ni maitatzen nauena izanen da maitatua ene Aitaz eta nik ere maitatuko dut haina eta agertuko diot ene burua.

39 - Jn $14,{ }^{23}$ Baldin nihork maitatzen banau ni, ene hitza begiratuko du $\varnothing$ eta ene Aitak maitatuko haina..

40 - Jn $17,{ }^{20} \mathrm{Ez}$ dut ... ordean horientzat xoilki otoitz egiten, bainan oraino hainentzat ere zeinek sinhetsiko baidute ene baitan, horien hitza aditurik. [horiek = ene dizipuluak]

\section{Harriet}

$\mathbf{1}$ - Mt, 6. ${ }^{2}$ Noiz ere emaiten baituzu amoina, ez dezazula adarra jo zure aitzinean, onarena egiten dutenek ohi duten bezala, bilkuetan eta karriketan gizonek goitituak izaitea gatik. Erraiten darotzuet egia, izana dute hainek bere golardoa. - (cf. Hnd, 1)

2, 3 - Mt 10, ${ }^{37}$ Nork ere maiteago baitu aita edo ama ni baino, haina ez da enetzat on; eta nork ere maiteago baitu semea edo alaba ni baino, haina ez da enetzat gai. — (cf. Hnd 2, 3 \& Duv, 9)

4 - Mt 15, ${ }^{5}$ Nork ere erranen baitio bere aitari edo amari: “[...]", 6eta haina egon daitekela bere aita edo ama lagundu gabe... - (cf. Hnd, 4 \& Duv, 14)

5 - Mt 19, ${ }^{9}$ Nork ere utziko baitu bere emaztea, ezpada zeren bertze gizon batekin egon den, eta ezkonduko baita bertze emazte batekin, haina lohikerian datza; eta batek utzia bertze batek hartzen badu, hura ere lohikerian datza. - (cf. Duv, 19)

6 - Mt 19, ${ }^{11}$ Eztira ere oro gai horren aditzeko, bainan hainak bakarrik, zeinei emana izan baitzaie zerutik. - (cf. Hnd, 5)

7 - Mt 19, ${ }^{29}$ Eta nork ere utziko baititu bere etxea, edo bere anaiak... ene izena gatik, hainari ordain emanen zaio ehunetan bertze hainbertze - (cf. Hnd, $6 \&$ Duv, 20)

8 - Mt $20,{ }^{23}$ ordean ene eskuinean edo ezkerrean jartzea, etzait niri zuei emaitea; bainan hainek izanen dute, zeinei apaindu baitaroe ene Aitak. - (cf. Hnd, $7 \&$ Duv, 21)

9 - Mt 24, ${ }^{46}$ Dohatsua da zerbitzari hura, zeina nausiak, datorrenean, horrela hari dela aurkituko baitu. ${ }^{47}$ Egiaz erraiten darotzuet, haina bere ontasun guzien gaineko ezarriko duela. - (cf. Hnd, 11)

10 - Mc 4, ${ }^{18}$ Eta elhorrietan ereiten zaroenak, hainak dira zeinek entzuten baitute hitza (cf. Hnd, 13 \& Duv, 28)

11 - Mc 4, ${ }^{20}$ Azkenean, lur onean ereiten zaroenak hainak dira zeinek entzuten baitute hitza, eta hartzen... - (cf. Hnd, 14 \& Duv, 29)

12 - Mc 6, ${ }^{11}$ Eta zenbaitek ezpazaituztete hartu nahiko eta entzun, hainen ganik ilkitzean, iharros ezazue zuen oinetakoen herrautsa, lekukotasuntzat izan dadin hekien, kaltetan.

13 - Mc 7, ${ }^{11}$ Baldin norbaitek baderro aitari edo amari: "[...]", hainak legea bete duke.

14 - Mc 10, ${ }^{11}$ Nork ere bere emaztea uzten 
baitu, eta bertze bat hartzen, hainak lehen emazteri bere zuzena edekitzen dio.

15 - Mc 10, ${ }^{12}$ Eta emazte batek bere gizona uzten badu, eta bertze senhar batekin ezkon badadi, haina ere gaizki nahasten da

16 - Lc 12, ${ }^{47}$ sehi haina behinik bat, zeinak jakin izan baitu nausiaren nahia, eta ezpaita egon ernerik, eta ezpaitu egin haren nahiaren eredura, zehatua izanen da garrazki. - (cf. Hnd, 21)

17 - Lc 14, ${ }^{33}$ Hala beraz, nork ere zuetarik ezpaitaie uko egiten dituen guziei, haina ezin izan daite ene dizipulu. - (cf. Hnd, 22)

18 - Lc 17, ${ }^{1}$ Baitezpada gertatu beharrak dira gaiz-bide emaiteak; bainan zorigaitz gaizbiderik emanen duenari! ${ }^{2}$ Hobe lizate hainarentzat baldin ihara harri bat ezar balakio lepotik dilindan, eta itsas ondalerat igotz baladi, ezen ez ttipitto hautarik bati gaizki eragin balioza. - (cf. Hnd, $21 \&$ Duv, 45-46)

19 - Jn 1, ${ }^{12}$ Bainan hura hartu izan duten guziei, eman izan daroe ahala Jainkoaren seme izaiteko, hainei zeinek sinheste baitute haren izenean. - (cf. Hnd, 26 \& Duv, 5051)

20 - Jn 5, ${ }^{24}$ Ene hitza entzuten duenak, eta sinhesten duenak ni egorri nauena baitan, hainak badu bizitze betierekoa; eta ezta hilikan ere galtzen, bainan heriotzetik bizirat doha. - (cf. Hnd, 29)

21 - Jn 6, ${ }^{47}$ Egiazki diotsuet: Nitan sinheste duenak, hainak badu bizitze betierekoa.

22 - Jn 12,26 ... Ene sehitasunean jartzen bada norbait, haina altxatuko du ene Aitak. - (cf. Hnd, 35 \& Duv, 64)

23, 24 - Jn 20, ${ }^{23}$ Noren ere bekatuak barkaturen baititutzue, barkatzen zaizko hainari; eta norenak ere edukiko baititutzue, hainarenak edukiak dirade.

\section{Duvoisin}

1, 2 - Mt 5, ${ }^{19}$ Nork ere beraz manamendu horietarik xumeena baitu hautsiren eta halako irakaspena gizonei baitiote emanen, haina zeruetako erresuman xumeena deituren da; aldiz nork ere eginen eta irakatsiren baititu, haina izanen da zeruetako erresuman handi deitua.

3, 4- Mt 5, ${ }^{22}$ Nik derratzuet ordean [...] nork ere berriz, anaiari erranen baitio, Raka, haina biltzarraren meneko izanen dela; zeinak aldiz erranen baitu, Erhoa, haina ifernuko suaren jasankizun dela.
5 - Mt 7, ${ }^{21}$ bainan ene Aita zeruetan denaren nahia egiten duena, haina da sarturen zeruetako Erresuman.

6 - Mt $7,{ }^{24}$ Edozeinek beraz adi eta bete detzan ene hitz haukiek, haina, harriaren gainean bere etxea jarri duen gizon gurbilaren kide eginen dute.

7 - Mt 7, ${ }^{26}$ Eta edozeinek adi detzan ene solas haukiek eta bete ez, haina izanen da, gizon zoro, legarraren gainean etxea egiten duenaren iduriko.

8 - Mt 10, ${ }^{22}$ Bainan zeinek ere ihardukiren baitu bururaino, haina izanen da salbatua.

9 - Mt 10, ${ }^{37}$ nork ere bere aita edo ama ni baino maiteago baitu, hura ez da enetzat egoki; eta nork ere nitaz gainetik maite baitu bere semea edo alaba, haina ez da enetzat egoki. - (cf. Hnd, 2, 3 \& Hrt, 2, 3)

10 - Mt 10, ${ }^{41}$ Zeinak profeta bat, profeta delakoz baitu hartzen, hainak profeta saria izanen du; eta nork ere prestu bat, prestu delakoz hartzen baitu, harek prestu saria du izanen.

11 - Mt 11, ${ }^{27}$ Eta nihork ez du ezagutzen Aita Semeak baizik eta hainak zeinari Semeak nahi izan baitio ezagutarazi.

12 - Mt 12, ${ }^{50}$ Ezen nork ere egiten baitu ene Aita zeruetan denaren nahia, haina da ene anaia, eta arreba, eta ama.

13 - Mt 13, ${ }^{19}$ Nork ere entzuten baitu erresumako hitza eta izpirituz ez ardietsen, etsai gaixtoa heldu zaio eta badarama hainaren bihotzean eraina zena.

14 - Mt $15,{ }^{5} \ldots$ Nork ere erranen baitio aitari edo amari : "[...]", hainak ez du ohoratu beharko aita edo ama. - (cf. Hnd $4 \& \mathrm{Hrt}$, 4)

15 - Mt 16, ${ }^{25}$ Ezen nork ere nahiko baitu bere bizia begiratu, harek galduko du; aldiz nork ere ene ariaz galduko baitu bizia, hainak aurkituren du.

16 - Mt $18,{ }^{4}$ Nork ere beraz bere burua haurtto hunen heinera baitu apalduren, haina zeruetako erresuman daite handienik.

17 - Mt $18,{ }^{5}$ Eta nork ere ene izenean harturen baitu hulaxeko haurtto bat, hainak neroni nau hartzen.

18 - Mt 18, ${ }^{6}$ Aldiz nork ere ene baitan sinhesten duten xume haukietarik bati gaizbiderik emanen baitio, hainak hobe luke ihara harri bat lepotik ezar baliozote, eta itsasoaren zolan ito balezate.

19 - Mt 19, ${ }^{9}$ Nik derratzuet bada, edozeinek utz dezan bere emaztea, non ez bertzearekin 
kutsaku delakoz, eta bertze batekin ezkontzen [sic] dadien, haina linburtzen dela; eta utziarekin ezkontzen dena, hura ere linburtzen dela. - (cf. Hrt, 5)

20 - Mt $19{ }^{29}$ Eta nork ere ene izenaren ariaz utziko baititu etxea, edo anaiak ..., hainak ehunkun izanen... - (cf. Hnd, 5 \& Hrt, 7)

21 - Mt 20, ${ }^{23}$ jartzea ordean ene eskuinetarik eta ezkerretarik etzait niri ematea zuei, bainan hainei zeinentzat berezi baitu ene Aitak. - (cf. Hnd, 7 \& Hrt, 8)

22 - Mt21, ${ }^{44}$ Eta harri haren gainera eroriko dena, zatikaturen da; aldiz noren gainerat ere eroriko baita harri hura, lehertuko du haina. - (cf. Hnd, 10)

23 - Mt $24,{ }^{13}$ Nork ere azkeneraino ihardukiren baitu, haina izanen da salbatua.

24 - Mt 26, ${ }^{23}$ Jatekoan enekin eskua bustitzen duenak, hainak nau salduko.

25 - Mc 3, ${ }^{35}$ Edozeinek egin dezan Jainkoaren nahia, haina da ene anaia, eta ene arreba, eta ene ama.

26 - Mc 4, ${ }^{14}$ Eraileak, hitza eraiten du. ${ }^{15}$ Bide bazterrekoak, hainak dire zeinen baitan eraiten baita hitza. Eta aditu duteneko, berehala dator debrua, eta badarama hekien bihotzetan eraina zen hitza.

27 - Mc 4, ${ }^{16}$ Halaber, toki harritsuetara eraiten zaiotenak, hainak dire, zeinek aditza ditu bezain sarri, berehalakoan bozkarioz onhesten baitute. - (cf. Hnd, 12)

28 - Mc 4, ${ }^{18}$ Eta bertzeak, elorrietara eraiten zaioetenak, hainak dire zeinek entzuten baitute hitza... - (cf. Hnd, 13 \& Hrt, 10)

29 - Mc 4, ${ }^{20}$ Eta hainak dire lur onean erain zaiotenak, zeinek aditzen baitute hitza, eta onhesten, ... - (cf. Hnd, 147 \& Hrt, 11)

30 - Mc $8,{ }^{38}$ Ezen ... norbaitek nitaz eta ene hitzez ahalke hartzen badu, Gizonaren Semeak ere hainaz ahalke izanen du..

30A - Mc 9, ${ }^{37}[\ldots]$ Nausia, ikusi dugu norbait zure izenean debruak iraizten zituena, haina ez baita guri jarraikitzen, eta debekatu diogu.

31 - Mc $9{ }^{40}$ Ezen nork ere baso bat ur edatera ematen baitarotzue ene izenean, Kristorenak zaretelakoz, egiaz derratzuet ez duela hainak bere saria salduko.

32 - Mc $9,{ }^{41}$ Eta nor ere izanen baita nitan sinheste duten xumetto haukietarik batentzat gaizbide, hainak hobe luke, ihara harri bat lepotik estekaturik, itsasora artik balezate. (cf. Hnd, 15)

33 - Mc 10, ${ }^{40}$ Ene eskuinean edo ezkerrean jartzea, ez dago niri ematea; bainan toki hekiek hainentzat dire zeinentzat apain[d]uak baitire. - (cf. Hnd, 16)

34 - Mc 10, ${ }^{43}$ aitzitik nork ere nahiko baitu handiena izan, haina zuen zerbitzari beharko da jarri.

35 - Mc $12,{ }^{19}$ Norbeiten anaia hiltzera heldu bada, eta uzten badu emaztea eta haurrik ez, hainaren anaiak har beza haren emaztea, eta ondore eman biozo bereianaiari.

36 - Mc $14,{ }^{21}[\ldots]$ bainan zorigatiz gizon hari, zeintaz saldua izanen baita gizonaren semea; hainak hobe zukeen sortu ez balitz ere. (cf. Hnd, 17)

37 - Lc 6, ${ }^{34}$ Eta hainei, mailegutan ematen baduzue, zeinen ganik igurikitzen baituzue ordaina, zer izanen da zuen eskerra?

38 - Lc 6, ${ }^{47}$ Ene gana datorrena, ene hitzak entzun eta egiten dituena, haina noren iduriko den erakutsiko darotzuet:

39 - Lc 6, ${ }^{49}$ Aldiz aditzen duenak eta ez egiten, hainak iduri du, asenturik gabe lurraren gainean etxe egiten hari den gizona...

40 - Lc 8, ${ }^{15}$ Lur onerat eroriak [=erori diren bihiak] hainak dire zeinek bihotza on eta guziz onean hartu eta iduki baitute hitza..

41 - Lc $8{ }^{21}$ Ene ama eta anaiak, hainak dire zeinak Jainkoaren hitza entzuten eta betetzen baitute. - (cf. Hnd, 19)

42 - Lc 10, ${ }^{22}$ nihork ez daki nor den Semea, Aitak baizik; eta nor den Aita, baizik-ere Semeak eta hainak zeinari Semeak nahi izan baitio ezagutarazi.

43 - Lc 12, ${ }^{5}$ Erakutsiren darotzuet noren beldur izan behar zareten: Hainarentzat izan zaitezte beldur, zeinak hil dukeen ondoan, ifernurat artikitzeko ahala baitu.

44 - Lc $12^{8}$ Edozeinek aitor nezan gizonen aitzinean, Gizonaren Semeak ere aitorturen duela haina Jainkoaren aingeruen aitzinean.

45, 46 - Lc $17,{ }^{1}$ Gerta ditezen gaizbideak, ezinbertzea da; bainan zorigaitz hainari, zeinaren egitez gaizbideak gertatzen baitire. ${ }^{2}$ Hainak hobe luke, ihara harri bat lepotik ezar eta itsasora artik balezate, ezenez xumetto haukietarik gaizbidetan erorraraz baleza. - (cf. Hnd, 23 \& Hrt, 18)

47 - Lc 17, ${ }^{15}$ Bada, hekietarik batek ikusi zuenean bere burua garbitua, gibelerat egin zuen, goraki handietsiz Jainkoa. ${ }^{16} \mathrm{Eta}$ ahuspez erori zen Jesusen oinetara, eskerrak bihurtzen ziozkielarik; eta haina Samariarra zen. - (cf. Hnd, 24)

48, 49 - Lc $17,{ }^{33}$ Nor ere hariko baita bere 
biziaren salbatzera, hainak galduko du; eta nork ere galduko baitu, hainak du aurkituren.

50, 51 - Jn 1,12Bainan zenbatek ere onhetsi baitute, hainei eman diote Jainkoaren ume bilhakatzeko ahala, hainei zeinek haren izenean sinheste baitute. - (cf. Hnd, 26 \& Hrt, 19)

52 - Jn $1,{ }^{23} \mathrm{Ni}$ naiz hainaren mintzoa, zeinak mortuan oihu egiten baitu.

53 - Jn 1, ${ }^{30 \mathrm{Hau}}$ da haina, zeintaz erran baitut:

54 - Jn 1,33bainan urean bataiatzen haritzera bidali nauenak, hainak erran izan darot ....

55 - Jn $5,{ }^{11}$ Nork ere sendatu bainau, hainak erran izan darot :...

56 - Jn $5,{ }^{38}$ zeren nor ere bidali baitu, haina ez baituzue sinhesten.

57 - Jn 6, ${ }^{57}$ Ene haragia jaten eta ene odola edaten duena, ene baitan dago, eta ni haren baitan. ${ }^{58}$ Nola Aita bizi denak igorri bainau, eta ni bizi bainaiz Aitaren ariatik, haina ere ene ariatik biziko da.

58 - Jn 7, ${ }^{16}$ Ene irakasmena ez da enea, bainan ni bidali nauenarena. ${ }^{17}$ Hainaren nahia egin nahi badu norbaitek, ezaguturen du hean irakasmen hau Jainkoaren ganikakoa den, ala ene baitarik naizen mintzo.

59 - Jn $7,{ }^{18} \ldots$ nork ere ordean bilhatzen baitu bera igorri duenaren gorestea, Haina zin da, eta zuzen ez denik haren baitan ez da. - (cf. Hnd, 32)

$60-$ Jn $8,{ }^{51}$ Nork ere begiraturen baitu ene hitza, hainak ez du nihoiz-ere ikusiren herioa.

61 - Jn 9, 22 .. alabainan Juduak hitzartuak ziren orduko, baizik-eta nihork aitor bihurtzen bazuen Jesus Kristo zela, haina sinagogatik kanpo ezarria izanen zela. - (cf. Hnd, 33)

62 - Jn 10, ${ }^{1}$ Arditegian atetik sartzen ez dena, bainan bertze nonbaitik igaiten, haina ebasle eta ohoin bat da.

63 - Jn $10,{ }^{35}$ Baldin jainko deitu baditu hainak zeineri ateratu baitzaiote Jainkoaren hitza... - (cf. Hnd, 34)

64 - Jn $12,26 \ldots$ Baldin zerbitzatzen banau norbaitek, ohore hainari emanen dio ene Aitak. - (cf. Hnd, 35 \& Hrt, 22)

65 - Jn 13,24 ... Nortaz mintzo ote da? 25 ... Nor da, Jauna? ${ }^{26}$ Haina zeinari ogi bustia eskainiko baitiot. — (cf. Hnd, 36-37)

66 - Jn 15, ${ }^{5}$ Zein ere baitago nitan, eta ni hartan, hainak ekarriren du fruitu hainitz

\section{Références}

\section{Sources basques}

Duv: DUVOISIN, Jean. 1859-65. Bible edo Testament Zahar eta Berria [...]. Londres. Facsim., Bilbao: Gran Enciclopedia Vasca, 1972.

Hnd : HARANEDER, Joannes. 1742. Jesu Christoren Evangelio Saindua. Ed. par P. Altuna, Bilbao : Euskaltzaindia, 1990.

Hrt : HARRIET, M./J. [avec la participation de L. DASSANCE]. 1855. Iesu-Christo gure Iaunaren Testament Berria [...]. Bayonne: E. Lasserre.

LEON, L. 1946. Jesu-Kristo gure Jaunaren Ebanjelio Saindua. Ustaritz: Chez l'auteur.

LIZARRAGA, J. 1868. Jesucristoren Evangelio Saindua, Juanec dacarran guisara. Londres.
Rééd. in L.-L. Bonaparte, Opera Omnia Vasconice, III (Bilbao: Euskaltzaindia), 13102.

\section{Travaux linguistiques}

BACH, E., \& R. COOPER. 1978. 'The NP-S Analysis of Relative Clauses and Compositional Semantics. Linguistics \& Philosophy 2, 145-150.

BOSKOVIC, Z. 1997. 'Superiority Effects with Multiple Wh-Fronting in Serbo-Croatian'. Lingua 102, 1-20.

CHOMSKY, N. 1986. Konwledge of Language. New York: Praeger.

DAYAL, V. 1995. 'Quantification in Correlatives'. E. Bach et al. (eds.), Quantification in Natural Languages (Dordrecht: Kluwer), 179-205.

DAYAL, V. 1997. Locality in $W H$ 
Quantification. Questions and Relative Clauses in Hindi. Dordrecht: Kluwer.

HEIM, I. 1982. The Semantics of Definite and Indefinite Noun Phrases. Thèse, Univ. du Massachusetts à Amherst. Ed. commerciale: New York: Garland, 1989.

IZVORSKI, R. 1996. 'The Syntax and Semantics of Correlative Proforms'. NELS 26 (ed. par K. Kusamoto), 133-147.

KEENAN, E. 1985. 'Relative Clauses'. T. Shopen (ed.), Language Typology and Syntactic Description, II (Cambridge : CUP), 141-170.

ORTIZ DE URBINA, J. 1989. Parameters in the Grammar of Basque. Dordrecht: Foris

OYHARÇABAL, B. 1985. Les relatives en basque.Univ. Paris 7: Collection ERA 642.

OYHARÇABAL, B. 1987. Etude descriptive de constructions c omplexes en basque [...]. Thèse d'Eat, Univ. Paris 7.

PARTEE, B. 1987. 'Noun Phrase Interpretation and Type-Shifting Principles'. J. Groenendijk, D. de Jong \& M. Stockhof (eds.), Studies in Discourse Representation Theory and the Theory of Generalized Quantifiers Dorecht: Foris), 115-143.

REBUSCHI, G. 1997. 'Remarques sur le pronom haina'. Lapurdum 2, 63-81.

REBUSCHI, G. 1998. 'E-Type Pronouns and Quantification in a Basque Dialect'. Communication au Colloque de sémantique (de préférence non-lexicale), Univ. Paris VII, 28-30 mai. Ms, Univ. Paris III.

ZAEFFERER, D. 1990. 'Conditionals and Unconditionals in Universal Grammar and Situation Semantics'. R. Cooper et al. (eds.), Situation Theory and its Applications, I (Stanford, CA: CSLI).

ZRIBI-HERTZ, A. \& J.-F. HANNE. 1995.

'Pronoms, déterminants et relatives en bambara de Bamako'. Linguistique africaine $15,91-135$. 